\title{
Ultrasound Parameters of Umbilical Artery Blood Flow Are Associated with Amniotic Fluid and Umbilical Artery Concentrations of Erythropoietin and Oxidative Stress Injury
}

\author{
Jiewen Tao, JingWang, Weiqi Jiang, Qi Meng, and Mingjuan Xu \\ Changhai Hospital, Second Military Medical University, China \\ Correspondence should be addressed to Mingjuan Xu; xumjuan1@163.com
}

Received 26 March 2021; Revised 29 May 2021; Accepted 1 June 2021; Published 17 June 2021

Academic Editor: Songwen Tan

Copyright (c) 2021 Jiewen Tao et al. This is an open access article distributed under the Creative Commons Attribution License, which permits unrestricted use, distribution, and reproduction in any medium, provided the original work is properly cited.

\begin{abstract}
Intrauterine hypoxia is the most frequent adverse intrauterine condition that occurs under a variety of circumstances including preeclampsia, placental insufficiency, high-altitude pregnancy, and any inflammatory condition during pregnancy resulting from gestational diabetes or even maternal obesity. However, early diagnosis of intrauterine hypoxia is still a challenge. In this study, we comparatively analyzed the systolic to diastolic ratio (S/D), resistant index (RI), and pulse index (PI) of the umbilical artery $(\mathrm{UmA})$ and middle cerebral artery (MCA) blood flows obtained from 46 pregnant women with intrauterine hypoxia and 80 normal pregnant women at 28-31, 32-36, and 37-41 gestational weeks. Results found that the S/D, RI, and PI of UmA and MCA blood flows at 28-31, 32-36, and 37-41 gestational weeks were all increased in hypoxic fetuses than in normal fetuses $(P<0.05)$. The malondialdehyde (MDA) level was elevated but superoxide dismutase (SOD), glutathione peroxidase (GSH-Px), and catalase (CAT) activities were reduced in the UmA blood of pregnant women with intrauterine hypoxia compared with normal pregnant women $(P<0.05)$. It was found that the NADPH oxidase $2($ Nox 2$)$ and NADPH oxidase 4 (Nox4) activities were increased in the UmA blood of pregnant women with intrauterine hypoxia compared with normal pregnant women $(P<0.05)$. Results of ELISA methods showed that the expression level of survivin was lower but the expression levels of caspase-3, caspase6 , and caspase- 9 were higher in the placental tissues of pregnant women with intrauterine hypoxia than those in normal pregnant women $(P<0.05)$. The concentrations of erythropoietin in the amniotic fluid and UmA blood were increased in pregnant women with intrauterine hypoxia compared with normal pregnant women $(P<0.05)$. The Spearman correlation analysis showed that the S/D, RI, and PI of UmA blood flow at 37-41 gestational weeks were positively correlated with the levels of Nox2, Nox4, and MAD and the UmA concentration of erythropoietin but negatively correlated with the activities of SOD, GSH-Px, and CAT $(P<0.05)$. In summary, the study indicates that ultrasound parameters of the UmA blood flow including $\mathrm{S} / \mathrm{D}, \mathrm{RI}$, and PI could serve as predictors of intrauterine hypoxia.
\end{abstract}

\section{Introduction}

There has been an increasing death rate of children younger than 5 years occurring in the neonatal period (aged $0-28$ days), which has resulted in much attention to neonatal mortality worldwide. The common causes of neonatal mortality refer to intrapartum-related factors such as hypoxic ischemic encephalopathy, preterm birth complications, infections including sepsis, meningitis, and neonatal tetanus, and other conditions including jaundice and congenital infections. Intrauterine hypoxia is the most frequent adverse intrauterine condition that occurs under a variety of circumstances including preeclampsia, placental insufficiency, highaltitude pregnancy, and any inflammatory condition during pregnancy resulting from gestational diabetes or even maternal obesity $[1,2]$ Accumulating evidence has showed that intrauterine hypoxia is associated with many fetal complications including low birth weight and developmental plasticity, resulting in indiscriminate damage to the developing fetus [3]. In several animal studies, intrauterine hypoxia could induce fetal growth restriction, cardiovascular dysfunction, and multiorgan morbidities of the fetus [4, 5]. Oxidative stress (OS) refers to excessive production of reactive oxygen species (ROS) and/or a decrease in antioxidant 
defense, where ROS overwhelms the ability of endogenous antioxidant systems to scavenge ROS [6]. Oxidative stress has implicated in the pathogenesis of hypoxia-induced disorders during intrauterine development and postnatal life, such as coronary artery disease, heart failure, hypertension, and type II diabetes [7-9]. Understanding the impact of intrauterine hypoxia and its generation of ROS during gestation is important for understanding the consequences of both fetal and neonatal outcomes. From this point of view, the earlier the diagnosis, the higher the plasticity and the more beneficial the effects.

The umbilical cord is a nutrition exchange channel between the fetus and the mother. Evaluation of its blood flow can predict the state of fetal intrauterine hypoxia and the severity of the disease. At present, there is no gold standard for the diagnosis of fetal intrauterine hypoxia. In clinical practice, comprehensive judgment is usually made through a variety of methods, such as fetal heart rate monitoring and fetal movement count, but the accuracy is limited. Although the pathogenesis of intrauterine hypoxia remains unclear and thus management and treatment are limited, it has been reported that fetoplacental blood vessels are compromised upon intrauterine hypoxia. Doppler ultrasound can detect the hemodynamic changes of umbilical artery (UA) and middle cerebral artery (MCA) to judge the abnormal situation of fetal organs, which is the main examination method for clinical noninvasive, timely, and accurate diagnosis of fetal intrauterine hypoxia. The resistance index (RI) and pulsation index (PI) of the fetal arterial blood flow impedance were measured, the peak systolic velocity of the diastolic velocity ratio (S/D) can fully reflect the peripheral circulation impedance and blood perfusion of the supplied vessels. Severe reduction in UmA blood flow as reflected by the absent or reverse end diastolic velocity during pregnancy is related to fetal morbidity and mortality, and early delivery should be contemplated to prevent intrauterine hypoxia-induced disorders [10]. High flow resistance in the capillaries of the terminal villi contributes to a low end-diastolic velocity in the UmA and subsequent hypoxia [11]. Herein, the present study analyzed S/D, RI, and PI of UmA and MCA blood flows obtained from 46 pregnant women with intrauterine hypoxia and normal pregnant women at 28-31, 32-36, and 37-41 gestational weeks and further explored their correlations with oxidative stress.

\section{Materials and Methods}

2.1. Study Subjects and Baseline Demographic Variables. This retrospective study included 46 pregnant women with intrauterine hypoxia and 80 normal pregnant women who delivered their babies in our hospital from January 2019 to January 2020. The Apgar scores of newborns delivered by normal pregnant women were 8-10 one minute after birth, and those of newborns delivered by pregnant women with intrauterine hypoxia were 0-7. At the same time, the blood gas analysis of the cord blood was carried out immediately after delivery. Fetal intrauterine hypoxia was diagnosed according to the $\mathrm{pH}$ value of UmA blood $<7.2$ and Apgar scores $<7$ scores.
The pregnant women with intrauterine hypoxia had age ranging from 33 to 39 years, with an average age of $36.63 \pm$ 5.24 years and gestational age at delivery ranging from 36 to 41 weeks, with an average gestational age of $37.90 \pm 0.67$ weeks. Their body mass index (BMI) was $23.40 \pm 2.51$ $\mathrm{kg} / \mathrm{m}^{2}$, gravidities were $2.30 \pm 1.20$ times, and abortions were $0.32 \pm 0.09$. The volumes of amniotic fluid, the Apgar scores of newborns one minute after birth, and the immediate $\mathrm{pH}$ value of UmA blood after delivery of pregnant women with intrauterine hypoxia were $28.22 \pm 4.71 \mathrm{~mm}, 5.86 \pm 1.18$, and $7.08 \pm 0.05$, respectively.

The normal pregnant women had age ranging from 34 to 39 years, with an average age of $36.83 \pm 4.92$ years, and gestational age at delivery ranging from 37 to 40 weeks, with an average gestational age of $38.77 \pm 0.74$ weeks. Their BMI was $23.15 \pm 2.42 \mathrm{~kg} / \mathrm{m}^{2}$, gravidities were $2.22 \pm 1.18$ times, and abortions were $0.43 \pm 0.20$. The volumes of amniotic fluid, the Apgar scores of newborns one minute after birth, and the immediate $\mathrm{pH}$ value of UmA blood after delivery of the normal pregnant women were $45.93 \pm 6.11 \mathrm{~mm}, 9.14$ \pm 0.69 , and $7.30 \pm 0.04$, respectively.

Pregnant women complicated with malignant tumor, severe metabolic diseases, gestational diabetes mellitus and hypertension, fetal congenital malformations, and incomplete clinical data were excluded out of the study.

2.2. Ultrasound Evaluation of UmA and MCA Blood Flow. The pregnant women held their breath, and the fetus was in quiescent condition. Color Doppler Ultrasound (Voluson730, GE Company, USA) was employed to evaluate the systolic to diastolic ratio (S/D), resistant index (RI), and pulse index (PI) of UmA and MCA blood flows at 28-31, 32-36, and 37-41 gestational weeks, with the frequency set at 3.04.0 MHz. Each parameter was tested in triplicates to obtain the average. Ultrasound evaluation of UmA and MCA blood flows was evaluated by experienced doctors for many years.

2.3. Measurements. The UmA blood was collected immediately from after delivery and stored in $2-8^{\circ} \mathrm{C}$. After centrifugation at $3500 \mathrm{r} / \mathrm{min}$ for $10 \mathrm{~min}$, the upper serum was extracted. The malondialdehyde (MDA) level and superoxide dismutase (SOD), glutathione peroxidase (GSH-Px), and catalase (CAT) activities were measured in the serum by immunoradiometric assays using commercial available kits (Diagnostic Products Corp., Los Angeles, Calif., USA) according to the instructions provided by the manufacture. The activities of NADPH oxidase 2 (Nox2) and NADPH oxidase 4 (Nox4) were measured by enzyme-linked immunosorbent assay (ELISA) according to kit's instructions (R\&D, Minneapolis, MN, USA). The fresh placental tissues were obtained within $30 \mathrm{~min}$ after delivery and then rinsed with normal saline followed by removal of calcified tissues and large vessels. The expression level of survivin protein was examined by ELISA according to kit's instructions (Abcam, Cambridge, UK) and caspase-3, caspase-6, and caspase-9 proteins according to kit's instructions (R\&D, USA). The concentrations of erythropoietin in the UmA blood and amniotic fluid were measured by ELISA using a commercial available kit (R\&D, USA). 
TABle 1: The S/D, RI, and PI of UmA and MCA blood flows in normal and hypoxic fetuses at 28-31 gestational weeks.

\begin{tabular}{|c|c|c|c|c|c|c|c|}
\hline \multirow{2}{*}{ Group } & \multirow{2}{*}{$N$} & \multicolumn{3}{|c|}{$\mathrm{UmA}$} & \multicolumn{3}{|c|}{ MCA } \\
\hline & & $\mathrm{S} / \mathrm{D}$ & RI & PI & $\mathrm{S} / \mathrm{D}$ & RI & PI \\
\hline Normal & 80 & $2.79 \pm 0.48$ & $0.64 \pm 0.07$ & $1.01 \pm 0.17$ & $5.69 \pm 1.26$ & $0.78 \pm 0.04$ & $1.81 \pm 0.19$ \\
\hline Hypoxic & 46 & $3.55 \pm 0.44$ & $0.71 \pm 0.03$ & $1.25 \pm 0.12$ & $3.31 \pm 0.44$ & $0.67 \pm 0.04$ & $1.28 \pm 0.16$ \\
\hline$t$ & & 8.81 & 6.44 & 8.44 & 12.37 & 14.86 & 15.94 \\
\hline$P$ & & $<0.001$ & $<0.001$ & $<0.001$ & $<0.001$ & $<0.001$ & $<0.001$ \\
\hline
\end{tabular}

Note: UmA: umbilical artery; MCA: middle cerebral artery; S/D: systolic to diastolic ratio; RI: resistant index; PI: pulse index.

TABle 2: The S/D, RI, and PI of UmA and MCA blood flows in normal and hypoxic fetuses at 32-36 gestational weeks.

\begin{tabular}{lcccccc}
\hline Group & $\mathrm{N}$ & $\mathrm{S} / \mathrm{D}$ & $\mathrm{UmA}$ & \multicolumn{2}{c}{$\mathrm{MCA}$} \\
$\mathrm{RI}$ & $\mathrm{PI} / \mathrm{D}$ & $\mathrm{PI}$ \\
\hline Normal & 80 & $2.49 \pm 0.38$ & $0.61 \pm 0.06$ & $0.92 \pm 0.15$ & $5.71 \pm 1.53$ & $0.82 \pm 0.05$ \\
Hypoxic & 46 & $3.71 \pm 0.65$ & $0.73 \pm 0.05$ & $1.32 \pm 0.18$ & $3.51 \pm 1.25$ & $0.70 \pm 0.06$ \\
$t$ & & 13.31 & 11.46 & 13.38 & 8.29 & $1.77 \pm 0.26$ \\
$P$ & $<0.001$ & $<0.001$ & $<0.001$ & $<0.001$ & $<.04$ & $<0.001$ \\
\hline
\end{tabular}

Note: UmA: umbilical artery; MCA: middle cerebral artery; S/D: systolic to diastolic ratio; RI: resistant index; PI: pulse index.

TABle 3: The S/D, RI, and PI of UmA and MCA blood flows in normal and hypoxic fetuses at 37-41 gestational weeks.

\begin{tabular}{|c|c|c|c|c|c|c|c|}
\hline \multirow{2}{*}{ Group } & \multirow{2}{*}{$\mathrm{N}$} & \multicolumn{3}{|c|}{ UA } & \multicolumn{3}{|c|}{ MCA } \\
\hline & & $\mathrm{S} / \mathrm{D}$ & RI & PI & $\mathrm{S} / \mathrm{D}$ & RI & PI \\
\hline Normal & 80 & $2.21 \pm 0.34$ & $0.52 \pm 0.04$ & $0.97 \pm 0.17$ & $5.17 \pm 1.86$ & $0.71 \pm 0.04$ & $1.37 \pm 0.49$ \\
\hline Hypoxic & 46 & $3.34 \pm 0.42$ & $0.79 \pm 0.06$ & $1.31 \pm 0.40$ & $3.84 \pm 1.38$ & $0.60 \pm 0.05$ & $1.20 \pm 0.25$ \\
\hline$t$ & & 16.46 & 30.26 & 6.64 & 4.22 & 13.54 & 2.192 \\
\hline$P$ & & $<0.001$ & $<0.001$ & $<0.001$ & $<0.001$ & $<0.001$ & 0.03 \\
\hline
\end{tabular}

Note: UmA: umbilical artery; MCA: middle cerebral artery; S/D: systolic to diastolic ratio; RI: resistant index; PI: pulse index.

2.4. Statistical Analysis. SPSS 21.0 software (IBM, USA) was employed for data analysis. The measurement data were expressed as the mean \pm standard deviation, and the comparison between two groups was performed by $t$-test. The correlation analysis was performed by Spearman's test. $P<0.05$ was taken as statistically significant.

\section{Results}

3.1. Changes of Ultrasound Parameters of UmA and MCA Blood Flows in Hypoxic Fetuses. This retrospective study included 46 pregnant women with intrauterine hypoxia and 80 normal pregnant women. The two groups of pregnant women did not differ in terms of age, gestational age at delivery, BMI, gravidities, and abortion histories $(P>0.05)$; however, significant differences were found with regard to the volumes of amniotic fluid $(P=0.010)$, the Apgar scores of newborns one minute after birth $(P<0.001)$, and the immediate $\mathrm{pH}$ value of UmA blood after delivery $(P<0.001)$. It was observed that the S/D, RI, and PI of UmA blood flow at 28-31, 32-36, and 37-41 gestational weeks were all increased in hypoxic fetuses than in normal fetuses $(P<0.05)$. Furthermore, hypoxic fetuses exhibited higher
S/D, RI, and PI of MCA blood flow than normal fetuses $(P<0.05)$. More detailed data are listed in Tables $1-3$.

3.2. Serum Markers of Oxidative Stress in the UmA Blood of Pregnant Women with Intrauterine Hypoxia. Oxidative stress plays a crucial role in the pathogenesis of diseases related to hypoxia during intrauterine development and postnatal life, which is usually evaluated by expression levels of MDA, SOD, GSH-Px, and CAT. As shown in Figure 1, the MDA level was elevated but SOD, GSH-Px, and CAT activities were reduced in the UmA blood of pregnant women with intrauterine hypoxia compared with normal pregnant women $(P<0.001$, Table 4). Furthermore, it was found that the Nox 2 and Nox 4 activities were increased in the UmA blood of pregnant women with intrauterine hypoxia compared with normal pregnant women $(P<0.001$, Table 4$)$.

3.3. Expressions of Survivin and Caspase Proteins in the Placental Tissues of Pregnant Women with Intrauterine Hypoxia. Survivin and caspase are two common apoptotic factors, both of which have been recently reported to be associated with oxidative stress. We collected placental tissues from pregnant women within $30 \mathrm{~min}$ after delivery. Results 

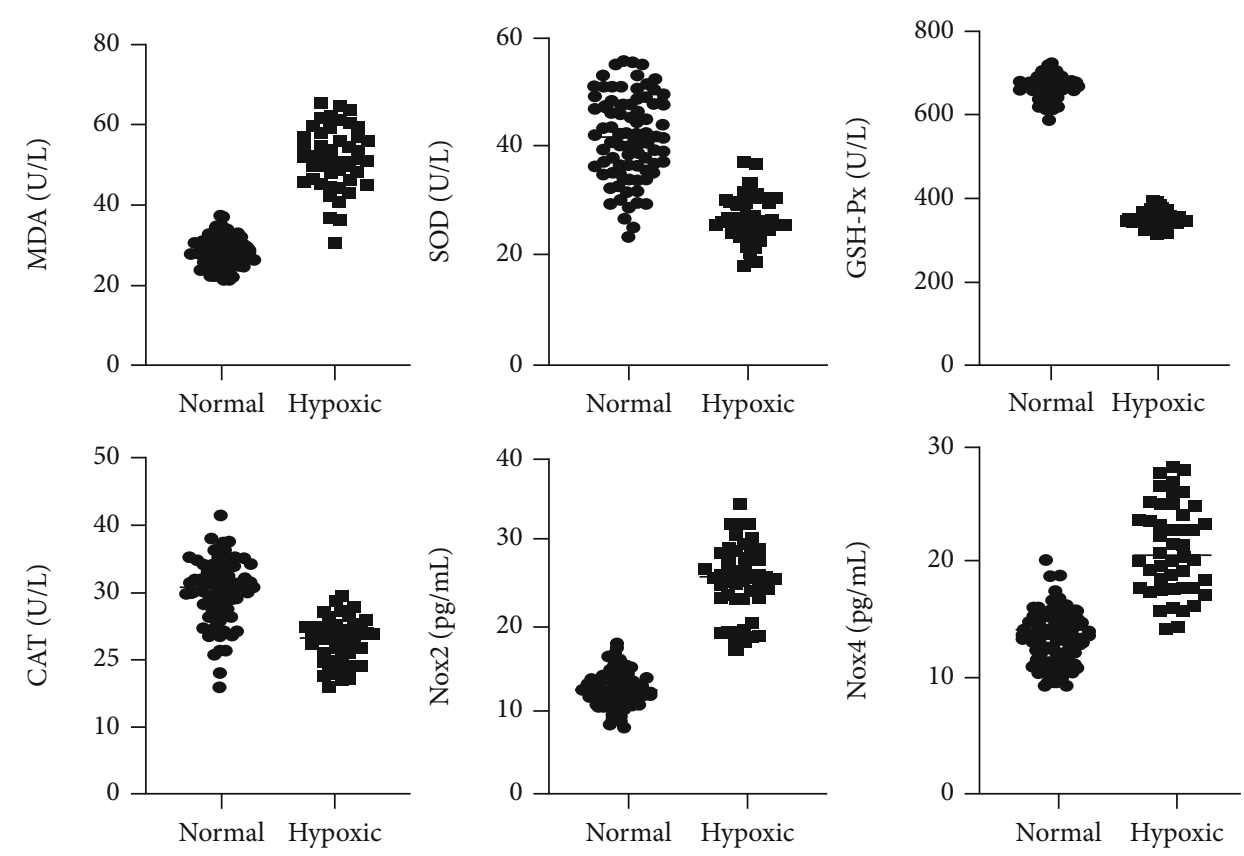

Figure 1: The levels of MDA, SOD, GSH-Px, CAT, Nox2, and Nox4 in the UmA blood between pregnant women with intrauterine hypoxia $(n=46)$ and normal pregnant women $(n=80)$.

TAble 4: The levels of MDA, SOD, GSH-Px, CAT, Nox2, and Nox4 in the UmA blood between pregnant women with intrauterine hypoxia and normal pregnant women.

\begin{tabular}{|c|c|c|c|c|}
\hline Marker & Normal $(n=80)$ & Hypoxic $(n=46)$ & $t$ & $P$ \\
\hline MDA (U/L) & $28.36 \pm 3.58$ & $51.49 \pm 7.80$ & 22.73 & $<0.001$ \\
\hline SOD (U/L) & $41.32 \pm 7.84$ & $26.70 \pm 4.41$ & 11.62 & $<0.001$ \\
\hline GSH-Px (U/L) & $664.76 \pm 27.52$ & $348.3 \pm 20.23$ & 68.08 & $<0.001$ \\
\hline CAT (U/L) & $30.24 \pm 4.83$ & $22.75 \pm 3.29$ & 9.338 & $<0.001$ \\
\hline Nox2 (pg/mL) & $12.51 \pm 2.02$ & $25.32 \pm 4.17$ & 23.19 & $<0.001$ \\
\hline Nox4 (pg/mL) & $13.61 \pm 2.25$ & $21.14 \pm 3.84$ & 13.90 & $<0.001$ \\
\hline
\end{tabular}

Note: MDA: malondialdehyde; SOD: superoxide dismutase; GSH-Px: glutathione peroxidase; CAT: catalase; Nox2: NADPH oxidase 2; Nox4: NADPH oxidase 4.

of ELISA methods showed that the expression level of survivin was lower but the expression levels of caspase-3, caspase6 , and caspase- 9 were higher in the placental tissues of pregnant women with intrauterine hypoxia than those in normal pregnant women $(P<0.001$, Figure 2$)$.

\subsection{Amniotic Fluid and UmA Concentrations of} Erythropoietin in Intrauterine Hypoxia. In adults and fetuses, erythropoietin is a glycoprotein that regulates erythropoiesis. Its production is mainly stimulated and regulated by tissue hypoxia. Chronic intrauterine hypoxia caused by various stimuli can cause the increase of erythropoietin. It was found by ELISA methods that the concentrations of erythropoietin in the amniotic fluid and UmA blood were increased in pregnant women with intrauterine hypoxia compared with normal pregnant women $(P<0.001$, Figure 3$)$.

3.5. Spearman Correlation between Ultrasound Parameters of UmA Blood Flow and Oxidative Stress in Intrauterine Hypoxia. We studied the correlation between ultrasound parameters of UmA blood flow and oxidative stress in intrauterine hypoxia. It was found that the S/D, RI, and PI of UmA blood flow at 37-41 gestational weeks were positively correlated with the levels of Nox2, Nox4, and MAD and the UmA concentration of erythropoietin but negatively correlated with the activities of SOD, GSH-Px, and CAT $(P<0.05$, Table 5).

\section{Discussion}

Intrauterine hypoxia can induce oxidative stress, which is not conducive to the growth and development of the fetus [12]. However, there is no effective way to prevent oxidative stress. Ultrasound parameters of UmA and MCA blood flows are important indicators for fetal physiological state and pathological injury. It was found that the S/D, RI, and PI of UmA and MCA blood flows at 28-31, 32-36, and 37-41 gestational weeks were all increased in hypoxic fetuses than in normal fetuses. The MDA, Nox2, and Nox4 levels were elevated but SOD, GSH-Px, and CAT activities were reduced in the 

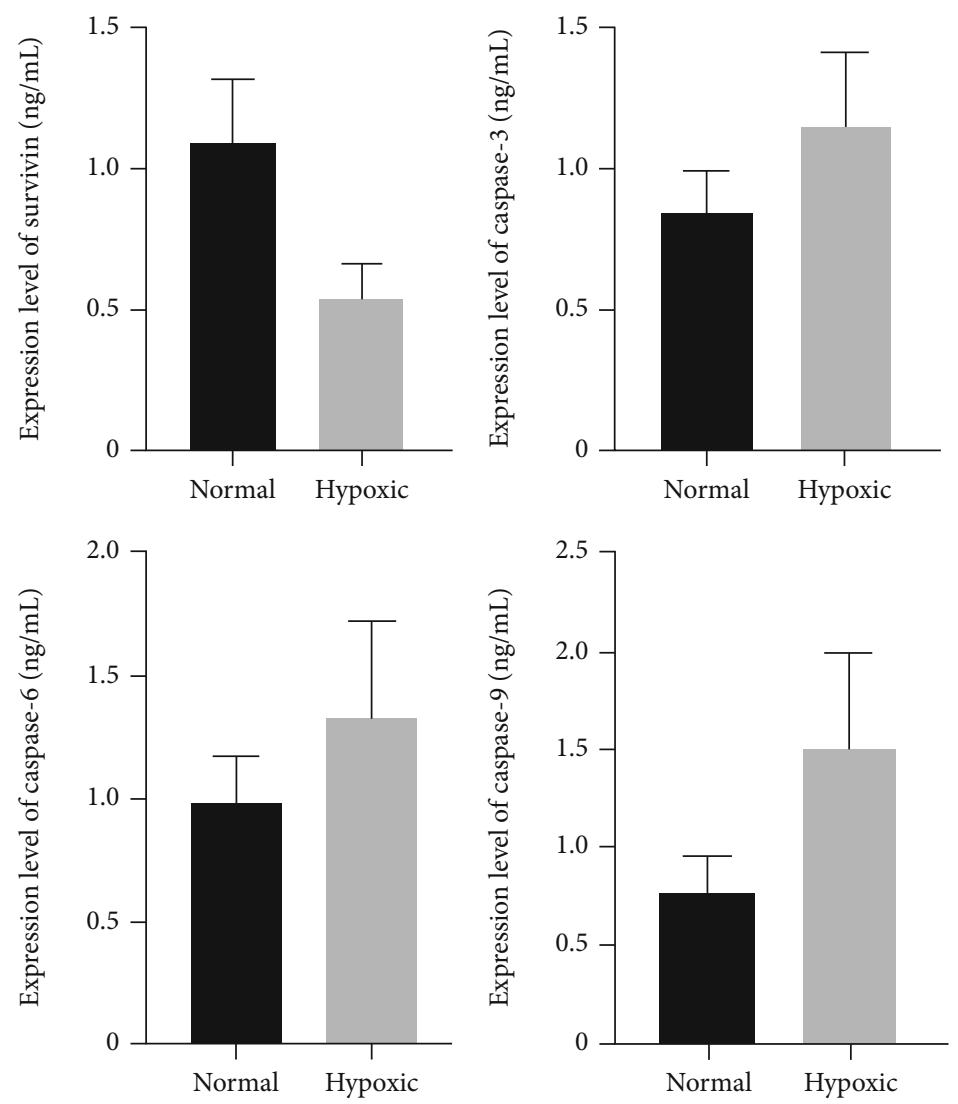

FIGURE 2: The expression levels of survivin, caspase-3, caspase-6, and caspase- 9 in the placental tissues between pregnant women with intrauterine hypoxia $(n=46)$ and normal pregnant women $(n=80)$. Results were expressed as the mean \pm standard deviation and analyzed by $t$-test.
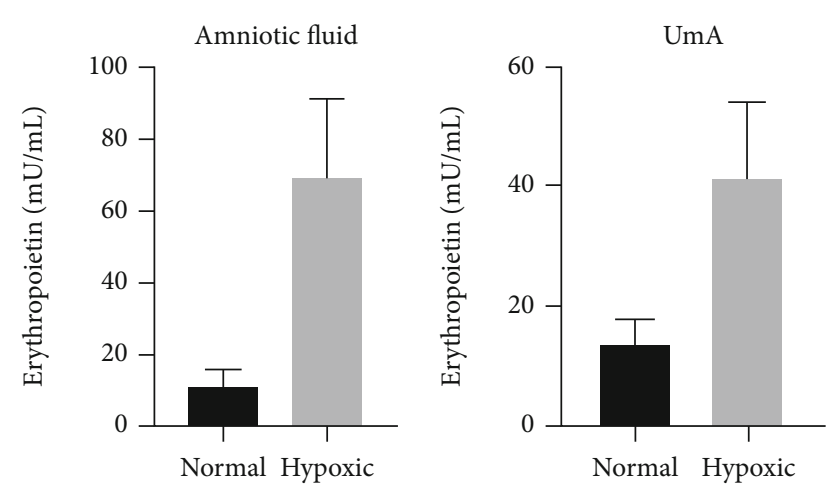

Figure 3: The concentrations of erythropoietin in the amniotic fluid and UmA blood between pregnant women with intrauterine hypoxia $(n=46)$ and normal pregnant women $(n=80)$. Results were expressed as the mean \pm standard deviation and analyzed by $t$-test.

UmA blood of pregnant women with intrauterine hypoxia compared with normal pregnant women. The expression level of survivin was lower but the expression levels of caspase-3, caspase- 6 , and caspase- 9 were higher in the placental tissues of pregnant women with intrauterine hypoxia than those in normal pregnant women. The concentrations of erythropoietin in the amniotic fluid and UmA blood were increased in pregnant women with intrauterine hypoxia compared with normal pregnant women. The Spearman cor- relation analysis showed that the S/D, RI, and PI of UmA blood flow at 37-41 gestational weeks were positively correlated with the levels of Nox2, Nox4, and MAD and the UmA concentration of erythropoietin but negatively correlated with the activities of SOD, GSH-Px, and CAT. All these findings indicate that the ultrasound parameters, S/D, RI, and PI, of UmA blood flow could reflect intrauterine hypoxia.

The UmA is the channel of material exchange between the fetus and the mother. The detection of UmA ultrasound parameters may have a reference value for prediction of fetal status [12]. Color Doppler ultrasound has the advantages of high safety, strong repeatability, and simple and convenient operation in the examination of the fetus, which can accurately predict the occurrence of fetal distress, and has high sensitivity and accuracy [13]. Gestational hypertension is a high-risk and frequently occurring disease in perinatal period. However, the pathogenesis of gestational hypertension is not completely clear at present. The main blood supply of the uterus is the uterine artery, which reflects the circulation of the uterus placenta. The monitoring of the fetal blood flow by color Doppler ultrasound in pregnant women with gestational hypertension can predict fetal oxidative stress to a certain extent [14]. Gestational diabetes mellitus can increase S/D value, which has adverse effects on fetal growth and development, suggesting that UmA blood flow parameters can be used as a reference index for fetal oxidative stress. Fetal hypoxia is the main cause of fetal distress that 
TABLE 5: Spearman correlation between ultrasound parameters of UmA blood flow at 37-41 gestational weeks, serum markers of oxidative stress, and concentrations of UmA erythropoietin in intrauterine hypoxia.

\begin{tabular}{lcccccc}
\hline \multirow{2}{*}{ Index } & \multicolumn{2}{c}{ S/D } & \multicolumn{2}{c}{ PI } & \multicolumn{2}{c}{ RI } \\
& $r$ & $P$ & $r$ & $P$ & $r$ & $P$ \\
\hline Nox2 & 0.512 & $<0.05$ & 0.385 & $<0.05$ & 0.441 & $<0.05$ \\
Nox4 & 0.667 & $<0.05$ & 0.424 & $<0.05$ & 0.475 & $<0.05$ \\
CAT & -0.395 & $<0.05$ & -0.411 & $<0.05$ & -0.482 & $<0.05$ \\
SOD & -0.221 & $<0.05$ & -0.377 & $<0.05$ & -0.398 & $<0.05$ \\
MDA & 0.349 & $<0.05$ & 0.386 & $<0.05$ & 0.452 & $<0.05$ \\
GSH-Px & -0.429 & $<0.05$ & -0.399 & $<0.05$ & -0.469 & $<0.05$ \\
Erythropoietin & 0.471 & $<0.05$ & 0.714 & $<0.05$ & 0.689 & $<0.05$ \\
\hline
\end{tabular}

S/D: systolic to diastolic ratio; RI: resistant index; PI: pulse index; MDA: malondialdehyde; SOD: superoxide dismutase; GSH-Px: glutathione peroxidase; CAT: catalase; Nox2: NADPH oxidase 2; Nox4: NADPH oxidase 4 .

mostly occurs after labor or pregnancy [15]. If intervention measures are not taken in time, neonatal asphyxia, neonatal neurological sequela, and neonatal hypoxic encephalopathy will occur and even endanger the life and health of the fetus. UmA blood flow parameters are an important means to monitor fetal distress [16]. Fetal heart rate monitoring combined with the umbilical cord blood flow S/D ratio monitoring has high application value in clinical prediction of fetal distress, which can effectively improve the diagnostic accuracy, take effective treatment measures in time, and improve fetal prognosis [17]. Ultrasound monitoring UmA combined with fetal heart rate monitoring is helpful to diagnose fetal distress, and the uterine artery blood perfusion of hypertension pregnant women is significantly higher than that of healthy pregnant women, and the fetal UmA blood flow resistance is higher, which affects the growth and development of the fetus [18]. This study also found that the RI and pulsation index of the pregnant women with intrauterine hypoxia were significantly higher than those of the normal pregnant women, suggesting that the RI and pulsation index of fetal UmA blood flow ultrasound parameters of pregnant women are closely related to oxidative stress.

The Nox protein family, CAT, SOD, and MDA are the main members involved in oxidative stress [19]. However, the existing evidence shows that ultrasound parameters play crucial roles in the judgment of oxidative stress as reflected by weakened right ventricular systolic function of the fetus in preeclampsia. The ultrasonic characteristics of the umbilical cord blood in the context of intrauterine hypoxia are increased resistance and decreased flow [10], which is closely related to the degree of oxidative stress and apoptosis of placental cells. The ultrasonic characteristics of the umbilical cord blood flow in patients with intrauterine hypoxia during the perioperative period are significantly different from those in normal pregnancy, which can provide the basis for clinical diagnosis of fetal intrauterine hypoxia. This study also found that the levels of CAT, SOD, and GSH-Px in the UmA blood of the pregnant women with intrauterine hypoxia were significantly lower than those of the normal pregnant women, while the levels of Nox2, Nox4, and MDA were significantly higher than those of the normal pregnant women. The S/D, RI, and PI of the fetal UmA blood of pregnant women with intrauterine hypoxia were significantly positively correlated with Nox2, Nox4 and MDA levels and significantly negatively correlated with CAT, SOD, and GSH-Px activities, suggesting that ultrasound parameters of the UmA blood flow were positively correlated with oxidative stress.

Hypoxia is commonly assigned a role in the placental dysfunction characteristic of preeclampsia and intrauterine growth restriction. Preeclampsia and intrauterine growth restriction are associated with increased apoptosis of placental villous trophoblast [20]. Survivin is a member of the inhibitor of apoptosis gene family that plays a key role in apoptosis inhibition and regulation of mitosis. It was reported previously that reduced expression of survivin was related with severity of preeclampsia [21]. Survivin can bind with caspase proteins to play an antiapoptotic role. As shown in our study, the expression level of survivin was lower but the expression levels of caspase-3, caspase-6, and caspase- 9 were higher in the placental tissues of pregnant women with intrauterine hypoxia than those in normal pregnant women.

Previous evidence showed that erythropoietin production is mainly stimulated and regulated by tissue hypoxia. Although the fetal kidneys are the main site of erythropoietin generation under basal conditions, recent experimental data indicate that the placenta is also an important site of erythropoietin generation in the circumstances of hypoxia [22]. Amniotic fluid erythropoietin levels have been shown to increase exponentially during fetal hypoxia in preeclamptic, diabetic, and $\mathrm{Rh}$-immunized pregnancies, which are correlated inversely with cord blood $\mathrm{pH}, \mathrm{pO}_{2}$, and base excess, and could serve as a predictor of neonatal morbidities and neonatal intensive care unit admission [23]. In this study, it was found by ELISA methods that the concentrations of erythropoietin in the amniotic fluid and UmA blood were increased in pregnant women with intrauterine hypoxia compared with normal pregnant women. As an indicator of chronic intrauterine hypoxia, fetal erythropoietin measurements have increased our knowledge about the pathogenesis and importance of intrauterine growth restriction, abnormal fetal heart rate, and abnormal Doppler flow patterns. While the clinical application of fetal amniotic fluid erythropoietin and importance of intrauterine growth measurements in the management of high-risk pregnancies and their offspring is promising, adequately powered clinical trials are urgently warranted.

These findings obtained from the present study support the notion that ultrasound parameters of UmA blood flow including $S / D$, RI, and PI could serve as predictors of intrauterine hypoxia. In the future research, we will further work from the following aspects: increasing the sample size of the study and using multicenter comparative study and exploring the correlation between ultrasound parameters of UmA and MCA blood flows and blood gas.

\section{Data Availability}

The data used to support the findings of this study are included within the article. 


\section{Conflicts of Interest}

All authors declare that they have no conflict of interest.

\section{Authors' Contributions}

Jiewen Tao and JingWang contributed to the work equally and should be regarded as co-first authors.

\section{References}

[1] P. Zhang, J. Ke, Y. Li et al., "Long-term exposure to high altitude hypoxia during pregnancy increases fetal heart susceptibility to ischemia/reperfusion injury and cardiac dysfunction," International Journal of Cardiology, vol. 274, pp. 7-15, 2019.

[2] K. L. Brain, B. J. Allison, Y. Niu et al., "Intervention against hypertension in the next generation programmed by developmental hypoxia," Plos Biology, vol. 17, no. 1, p. e2006552, 2019.

[3] P. J. Gonzalez-Rodriguez, F. Xiong, Y. Li, J. Zhou, and L. Zhang, "Fetal hypoxia increases vulnerability of hypoxicischemic brain injury in neonatal rats: role of glucocorticoid receptors," Neurobiology of Disease, vol. 65, pp. 172-179, 2014.

[4] C. F. Rueda-Clausen, V. W. Dolinsky, J. S. Morton, S. D. Proctor, J. R. Dyck, and S. T. Davidge, "Hypoxia-induced intrauterine growth restriction increases the susceptibility of rats to high-fat diet-induced metabolic syndrome," Diabetes, vol. 60, no. 2, pp. 507-516, 2011.

[5] M. Keenaghan, L. Sun, A. Wang, E. Hyodo, S. Homma, and V. S. Ten, "Intrauterine growth restriction impairs right ventricular response to hypoxia in adult male rats," Pediatric Research, vol. 80, no. 4, pp. 547-553, 2016.

[6] J. G. Farias, E. A. Herrera, C. Carrasco-Pozo et al., "Pharmacological models and approaches for pathophysiological conditions associated with hypoxia and oxidative stress," Pharmacology \& Therapeutics, vol. 158, pp. 1-23, 2016.

[7] K. L. Thornburg, P. F. O'Tierney, and S. Louey, "Review: the placenta is a programming agent for cardiovascular disease," Placenta, vol. 31, pp. S54-S59, 2010.

[8] D. A. Giussani, E. J. Camm, Y. Niu et al., "Developmental programming of cardiovascular dysfunction by prenatal hypoxia and oxidative stress," PLoS One, vol. 7, no. 2, p. e31017, 2012.

[9] E. A. Herrera, B. Krause, G. Ebensperger et al., "The placental pursuit for an adequate oxidant balance between the mother and the fetus," Frontiers in Pharmacology, vol. 5, p. 149, 2014.

[10] A. Jurisic, Z. Jurisic, E. Lefkou, J. Pombo, and G. Girardi, "Pravastatin and-L-arginine combination improves umbilical artery blood flow and neonatal outcomes in dichorionic twin pregnancies through an nitric oxide- dependent vasorelaxant effect," Vascular Pharmacology, vol. 110, pp. 64-70, 2018.

[11] A. Ebrashy, O. Azmy, M. Ibrahim, M. Waly, and A. Edris, "Middle cerebral/umbilical artery resistance index ratio as sensitive parameter for fetal well-being and neonatal outcome in patients with preeclampsia: case-control study," Croatian Medical Journal, vol. 46, no. 5, pp. 821-825, 2005.

[12] C. F. Rueda-Clausen, J. S. Morton, G. Y. Oudit, Z. Kassiri, Y. Jiang, and S. T. Davidge, "Effects of hypoxia-induced intrauterine growth restriction on cardiac siderosis and oxidative stress," Journal of Developmental Origins of Health and Disease, vol. 3, no. 5, pp. 350-357, 2012.

[13] Z. Fardiazar, S. Atashkhouei, Y. Yosefzad, M. Goldust, and R. Torab, "Comparison of fetal middle cerebral arteries, umbil- ical and uterin artery color Doppler ultrasound with blood gas analysis in pregnancy complicated by IUGR," Iranian journal of reproductive medicine, vol. 11, no. 1, pp. 47-51, 2013.

[14] L. Sarno, G. M. Maruotti, G. Saccone, M. Morlando, A. Sirico, and P. Martinelli, "Maternal body mass index influences umbilical artery Doppler velocimetry in physiologic pregnancies," Prenatal Diagnosis, vol. 35, no. 2, pp. 125-128, 2015.

[15] S. Raicevic, D. Cubrilo, S. Arsenijevic et al., "Oxidative stress in fetal distress: potential prospects for diagnosis," Oxidative Medicine and Cellular Longevity, vol. 3, no. 3, p. 218, 2010.

[16] T. Avitan, A. Sanders, U. Brain, D. Rurak, T. F. Oberlander, and K. Lim, "Variations from morning to afternoon of middle cerebral and umbilical artery blood flow, and fetal heart rate variability, and fetal characteristics in the normally developing fetus," Journal of Clinical Ultrasound, vol. 46, no. 4, pp. 235240, 2018.

[17] I. Aditya, V. Tat, A. Sawana, A. Mohamed, R. Tuffner, and T. Mondal, "Use of Doppler velocimetry in diagnosis and prognosis of intrauterine growth restriction (IUGR): a review," Journal of Neonatal-Perinatal Medicine, vol. 9, no. 2, pp. 117126, 2016.

[18] M. Tchirikov, M. Strohner, D. Forster, and B. Huneke, "A combination of umbilical artery PI and normalized blood flow volume in the umbilical vein: venous-arterial index for the prediction of fetal outcome," European Journal of Obstetrics, Gynecology, and Reproductive Biology, vol. 142, no. 2, pp. 129-133, 2009.

[19] S. Perrone, E. Laschi, and G. Buonocore, "Biomarkers of oxidative stress in the fetus and in the newborn," Free Radical Biology \& Medicine, vol. 142, pp. 23-31, 2019.

[20] C. F. Rueda-Clausen, J. L. Stanley, D. F. Thambiraj, R. Poudel, S. T. Davidge, and P. N. Baker, "Effect of prenatal hypoxia in transgenic mouse models of preeclampsia and fetal growth restriction," Reproductive Sciences, vol. 21, no. 4, pp. 492502, 2014.

[21] C. F. Li, W. L. Gou, X. L. Li, S. L. Wang, T. Yang, and Q. Chen, "Reduced expression of survivin, the inhibitor of apoptosis protein correlates with severity of preeclampsia," Placenta, vol. 33, no. 1, pp. 47-51, 2012.

[22] K. A. Teramo and J. A. Widness, "Increased fetal plasma and amniotic fluid erythropoietin concentrations: markers of intrauterine hypoxia," Neonatology, vol. 95, no. 2, pp. 105116, 2009.

[23] L. Seikku, L. Rahkonen, M. Tikkanen et al., "Amniotic fluid erythropoietin and neonatal outcome in pregnancies complicated by intrauterine growth restriction before 34 gestational weeks," Acta Obstetricia et Gynecologica Scandinavica, vol. 94, no. 3, pp. 288-294, 2015. 